\title{
François Mabille, Approches de l'internationalisme catholique
}

Paris, L'Harmattan, 2001, 241 p. (coll. « Religions et relations internationales »)

\section{Clémence Bosselut}

\section{(2) OpenEdition}

Journals

Édition électronique

URL : http://journals.openedition.org/assr/2234

DOI : $10.4000 /$ assr.2234

ISSN : $1777-5825$

Éditeur

Éditions de l'EHESS

Édition imprimée

Date de publication : 1 avril 2004

Pagination : 47-112

ISBN : 2-222-96746-5

ISSN : 0335-5985

Référence électronique

Clémence Bosselut, «François Mabille, Approches de l'internationalisme catholique », Archives de sciences sociales des religions [En ligne], 126 | avril - juin 2004, document 126.19, mis en ligne le 17 novembre 2005, consulté le 22 septembre 2020. URL : http://journals.openedition.org/assr/2234 ; DOI : https://doi.org/10.4000/assr.2234 
conséquence intellectuelle majeure l'impossibilité de se pencher «sur l'institutionnalité du langage et l'ordre dogmatique du sujet » (p. 113). Sur le plan théorique règne la confusion la plus totale : ainsi confond-on allègrement « sujet» et « individu». Le langage inaugure la déchosification et introduit le sujet dans la représentation, y compris par rapport à luimême, comme par rapport à son propre corps. Le sujet est divisé par le langage. Tout un chacun est dans une relation spéculaire à soi, à travers le thème du miroir : d'un côté, le corps physique, de l'autre, l'image. La société également fonctionne comme un miroir à l'égard du sujet. La relation spéculaire ne se limite pas cependant à assurer l'adhésion du sujet à sa propre image, elle introduit la problématique de l'altérité, puisque l'image est autre que celui qui se tient de ce côté-ci du miroir. Si cet autre peut devenir soi, alors tout autre peut devenir semblable dès lors qu'il peut être identifié à cette image. L'acquisition des mécanismes linguistiques par l'enfant reproduit ce processus, notamment l'utilisation de ce que R. Jakobson appelait shifters.

P.L. donne des éléments pour une critique $\mathrm{du}$ discours scientifique, notamment s'agissant de la confusion entre vérité scientifique et vérité dogmatique, ou ce que, en d'autres termes, on pourrait appeler la transformation des vérités scientifiques en valeurs. "Une vérité scientifique est scientifique, parce que conforme aux lois de la science considérée. Mais, dès lors que la société la reconstruit comme valeur, elle prend statut de vérité dogmatique. Le discours scientifique obéit aussi à la logique des montages institutionnels du discours » (p. 19). L'idée que, en donnant la primauté à la Science la civilisation occidentale s'est détachée de toutes celles qui l'ont précédée, est une croyance largement répandue. C'est aussi le fond du thème nietzschéen de « la mort de Dieu ». Ce que conteste fortement P.L., pour lequel les Occidentaux pas plus que les autres ne peuvent s'affranchir de la condition humaine: «1'Explication scientifique a pris valeur dogmatique...ce qui replace l'Occident ultramoderne dans la condition ordinaire des civilisations » (p. 178).

Hocine Benkheira.

MABILLE (François).

Approches de l'internationalisme catholique. Paris, L'Harmattan, 2001, 241 p. (coll. « Religions et relations internationales »).

Comprendre la place du catholicisme dans les relations internationales sécularisées est loin d'être un sujet nouveau pour F.B. En 1993, il a analysé le récent corpus doctrinal de l'Église catholique sur les relations internationales en en présentant les principales spécificités (L'Église et la paix. Vingt-cinq ans de messages pontificaux pour la paix. Centurion, 1993 [cf. Arch. 94.129]). En 2001, son Expertise internationale $d u$ catholicisme français (L'Harmattan, 2001) visait à comprendre la participation des catholiques à la production d'idées et à la mise en place des mobilisations collectives sur le plan international. L'ouvrage était fondé sur l'étude d'un acteur fonctionnant en réseau : les Commissions Justice et Paix aux niveaux nationaux et au niveau du Saint-siège. Par ailleurs, il publiera bientôt Les catholiques français et la paix au temps de la guerre froide (L'Harmattan, 2004), centré sur le mouvement international Pax Christi. Il y analyse le processus de l'internationalisation du catholicisme dans un contexte global de crise du catholicisme, notamment à travers les notions de sécularisation interne, de laïcisation et de pluralisation interne.

La longue introduction (de nature politiste) de Approches de l'internationalisme catholique permet à l'auteur de définir l'Église en tant qu'elle est à la fois acteur producteur d'idées, de normes, de socialisation, mais également acteur dans des situations conflictuelles. Il relate les évolutions des individualités, des collectifs nationaux ou internationaux et des ONG catholiques tout au long du $\mathrm{XX}^{\mathrm{e}}$ siècle. Le projet de «paix catholique » est placé au cœur de cette évolution de l' «internationalisme catholique ». L'A. situe ce projet dans le contexte de ce siècle, avec l'ambition de faire ressortir les constantes mais également la complexité des relations entre Église, État et consciences individuelles.

La première partie offre une typologie de l'internationalisme catholique, que l'on pourra comparer à celle de Jacques Gadille (1981). Elle donne des clés de compréhension simples et riches de l'évolution de sa stratégie au $\mathrm{XX}^{\mathrm{e}}$ siècle sur le plan international et un fil de lecture unificateur pour cet ouvrage. Les nombreuses références aux sources historiques en rendent la lecture d'autant plus intéressante.

La particularité de l'ouvrage est d'aborder ce sujet sur le plan thématique, sous la forme de monographies d'associations et mouvements français centrés sur la paix, les droits de l'homme, la fraternité, le développement et la coopération.

Le discours chrétien de mobilisation contre la torture est ainsi présenté, dans la seconde partie, dans son évolution historique. Notons 
que l'A. place ce discours au sein des problématiques des droits de l'Homme et du sentiment de solidarité internationale. Il met également à jour la relation entre la révolte des chrétiens contre la torture et l'évolution de la morale, en particulier à travers l'émancipation progressive des consciences à l'égard de l'État comme à l'égard de l'Église.

Dans une troisième partie, l'A. s'interroge sur le lien que fait Pax Christi entre paix et développement. Il situe dans ce chapitre l'émergence du thème du développement dans le discours de ce mouvement, à la fois sur le plan historique et sur le plan structurel. En les replaçant dans la conjoncture internationale de la guerre froide, il montre l'élargissement de la notion de "prochain » à celle d' "étranger », l'apparition de la thématique du développement liée à celle de la paix, et l'importance que le Mouvement lui donne dans le cadre d'une théologie du développement.

Un travail sur l'évolution du mouvement d'éducation populaire Vie Nouvelle permet ensuite d'aborder le thème de la fraternité. L'A. en décrit la progressive sécularisation. Ce thème de la fraternité, très présent dans la culture catholique, se trouve à la conjonction du religieux et du politique. Il constitue, pour l'A., l'essence de la solidarité internationale et est significatif des transformations de la relation de Vie Nouvelle à l'ouverture internationale.

La dernière partie de l'ouvrage revisite la notion de laïcité à travers l'exemple de la Délégation Catholique pour la Coopération (DCC). Cette problématique de la laïcité est particulièrement intéressante, au regard des débats actuels. L'A. montre comment ce concept peut être élargi : avec la DCC, on a un exemple d'organisation catholique créée à la demande de l'État, financée en partie par lui, puis, sur son intervention, constituée en association loi 1901. La DCC se trouve donc de fait au cœur de conflits et de négociations entre l'État et l'Église de France. Ici, le régime de séparation n'est donc pas celui de l'ignorance, mais celui de nouveaux types de relations, qui demandent d'ailleurs à être constamment redéfinis.

La méthodologie de l'A. est essentiellement historique, mais elle ne néglige pas pour autant les apports de la sociologie, de la sémantique et des sciences politiques. L'aspect sémantique est particulièrement riche et pertinent. Les mots sont toujours replacés dans leur acception de l'époque, à partir d'un travail sur les archives ; l'évolution de leur sens est souvent au cœur de la démonstration.
Cet ouvrage présente donc plusieurs intérêts : l'interdisciplinarité, les nombreuses citations et commentaires d'archives, l'apport réel de connaissances, la présentation de plusieurs mouvements et associations représentatifs du catholicisme d'hier et d'aujourd'hui. Cela montre l'ampleur du sujet abordé. L'aspect interdisciplinaire n'est bien sûr pas achevé ici : on peut d'emblée y voir une invitation à approfondir ces approches en vue de compléter cette réflexion sur un sujet relativement peu étudié.

Clémence Bosselut.

MARTIAL (Agnès).

S'APPARENTER. Ethnologie des liens de familles recomposées. Paris, Éditions de la Maison des Sciences de 1'Homme, 2003, 309 p. (bibliogr., annexes) (coll. «Ethnologie de la France »).

A.M. étudie dans cet ouvrage la manière dont la répartition des rôles parentaux et beauxparentaux s'effectue dans les configurations familiales recomposées. La diversité et l'hétérogénéité des situations qui ont constitué son terrain, lui ont offert un éventail de parcours biographiques à partir desquels elle explore la signification des liens de familles recomposées. À partir des éléments récurrents de ces récits émergent des thèmes cristallisant les questions relatives à la façon dont chacun vit et se représente ou non comme un «parent » de sang ou de fait, dans les familles recomposées.

Elle aborde ainsi successivement les statuts générationnels, l'interdit de l'inceste, la parentalité, la succession, l'adoption, autant d'éléments liés à la parenté dans le contexte de la recomposition familiale.

À l'incertitude des statuts et des rôles, par exemple lorsque la recomposition met en présence un jeune beau-père et sa belle-fille adulte, s'ajoute le constat de la vulnérabilité des liens familiaux recomposés. La tendance à la dissolution des liens paternels interroge la définition du rôle paternel. La fragilité des liens père-enfant s'aggrave au fur et à mesure que l'on descend dans l'échelle sociale. L'affaiblissement du rôle paternel n'est pas compensé par la présence d'un beau-père qui veille le plus souvent à ne pas déborder les limites de son rôle « additionnel». Il y a une tendance dominante à la «monoparentalité éducative» au sein de familles que l'on peut définir comme «matricentrées ». Se dessine le risque de voir les enfants «désaffiliés » d'une partie de leurs origines et de leur identité familiales. C'est alors le groupe social tout entier, à travers 